DOI: https://doi.org/10.11144/Javeriana.upsy18-3.cpeu

\title{
Conducta prosocial en estudiantes universitarios argentinos: estudio comparativo entre carreras*
}

Prosocial Conduct in Argentinian University Students: Comparative Study between Careers

Recepción: 12 Abril 2018 | Aceptación: 19 Julio 2019

\author{
Sofía Esmeralda Aunéa \\ Consejo Nacional de Investigaciones Científicas y \\ Técnicas (CONICET), Argentina \\ ORCID: http://orcid.org/0000-0002-0620-0199 \\ Facundo Juan Pablo Abal \\ Consejo Nacional de Investigaciones Científicas y \\ Técnicas (CONICET), Argentina \\ ORCID: http://orcid.org/0000-0001-7023-5380
}

Horacio Félix Attorresi

Universidad de Buenos Aires, Argentina

ORCID: https://orcid.org/0000-0002-3027-1069

a Autor de correspondencia. Correo electrónico: sofiaaune@psi.uba.ar

Para citar este artículo: Auné, S. E., Abal, F. J. P., \& Attorresi, H. F. (2019). Conducta prosocial en estudiantes universitarios argentinos: estudio comparativo entre carreras. Universitas Psychologica, 18(3), 1-12. https://doi.org/10.11144/Javeriana. upsy18-3.cpeu

\section{RESUMEN}

En este estudio, se puso a prueba la hipótesis sobre las diferencias en conducta prosocial según el tipo de carrera estudiada. Se utilizó la Escala de Conducta Prosocial, un test previamente modelado con los procedimientos clásicos y con la Teoría de la Respuesta al Ítem, conformado por dos dimensiones: Ayuda y Confortar. Se midieron asimismo diferencias en la Autopercepción en Orientación Prosocial (AOP) y en Distorsión. Una muestra depurada de 1383 estudiantes universitarios de diferentes carreras se dividió teniendo en cuenta si la carrera involucra o no directamente a las conductas prosociales, y en caso afirmativo, distinguiendo si lo hace en relación con la salud física. La media de edad fue de 22.4 años, ocupando las mujeres el $79 \%$ de la muestra. Los resultados indicaron que los estudiantes de carreras que involucran conductas prosociales relacionadas con la salud física puntuaron significativamente más alto en la dimensión Ayuda que el resto, aunque igualmente tuvieron más distorsión en su respuesta. En cuanto a la dimensión Confortar, los estudiantes de carreras directamente vinculadas con las conductas prosociales en general fueron quienes puntuaron más alto. Dentro de la AOP, el tipo de carrera tuvo efectos significativos sobre el puntaje en Autopercepción de la Conducta Prosocial y en Autopercepción de Dadivosidad. Se discuten los resultados y las limitaciones.

Palabras clave

conducta prosocial; estudiantes universitarios argentinos; ayuda; confortar; orientación prosocial.

\section{ABSTRACT}

This study tests the hypothesis of differences in prosocial behavior according to university studies using the Prosocial Behavior Scale, a test 
previously modeled through classical procedures and the Item Response Theory comprising two dimensions, Help and Comfort. Differences were also measured regarding self-perception in prosocial orientation (AOP) and distortion. A refined sample of 1383 university students of different courses was divided considering whether the course directly involves prosocial behavior and, if so, whether this behavior is associated to health. The mean age was 22.4 years, with women accounting for $79 \%$ of the sample. Results showed that students in courses involving prosocial behavior related to health scored significantly higher than the rest of the group in the Help dimension, although they also exhibited higher response distortion. Regarding the Comfort dimension, students in courses directly linked to prosocial behavior in general rendered the highest scores. The course of studies also had significant effects on AOP and self-perceived generosity. Results and limitations are discussed.

Keywords

prosocial behavior; Argentinian university students; help; university comfort; prosocial orientation.

Los avances en la investigación psicológica responden a condiciones derivadas de la relación compleja entre cuestiones históricas y epistemológicas (Macbeth, Cortada de Kohan, Razumiejczyk, \& López Alonso, 2006). En este sentido, a lo largo de su historia, la psicología se ha concentrado mucho más frecuentemente en las emociones negativas y las patologías mentales. Específicamente, después de la Segunda Guerra Mundial, las investigaciones se concentraron en el estudio de los desórdenes mentales, ya que muchas personas habían sido afectadas por la guerra y la incidencia de patologías se había convertido en un problema acuciante (Seligman, Parks, \& Steen, 2004). Sin embargo, en los últimos años, los investigadores comenzaron a interesarse en el bienestar subjetivo, siendo la llamada psicología positiva un área en crecimiento (Donaldson, Dollwet, \& Rao, 2015) ya que se empezó a concebir que la ausencia de malestar no es igual a felicidad (Seligman et al., 2004). Se podría estar en presencia de un cambio de paradigma, un cambio de gran alcance que implica una forma diferente de concebir la realidad (Macbeth, Cortada de Kohan, \& Razumiejczyk, 2007). La psicología positiva es el marco necesario para realizar investigaciones que promuevan calidad de vida y bienestar (Mikulic et al., 2006; Seligman \& Csikszentmihalyi, 2014). Además, en lo social, se destaca el creciente énfasis de los nuevos desarrollos psicológicos (Macbeth et al., 2006).

Los actos orientados a los otros parecen ser una parte esencial y única de la vida social humana, siendo objeto de estudio de la psicología positiva. Para Marean (2015), la tendencia a cooperar incluso con individuos no emparentados supuso el logro de conexiones sociales flexibles. Esta fue una de las claves para la supervivencia y la hegemonía del Homo Sapiens sobre otros Homos. Para este autor, existiría incluso una predisposición genética que facilita este tipo de conductas.

Es así como la conducta prosocial, es decir, toda conducta social positiva con o sin motivación altruista (González Portal, 2000) es uno de los constructos centrales estudiados por la psicología positiva. Ningún acto es inherentemente prosocial, sino que esto debe ser evaluado en el contexto social y cultural específico donde ocurre la acción (Schroeder \& Graziano, 2015). Se ha conceptualizado a dicha conducta como un fenómeno complejo que involucra acciones de los individuos basadas en creencias y sentimientos, y que describe la forma en que estos se orientan hacia los otros al realizar conductas solidarias (Auné, 2018).

La realización de conductas prosociales aumenta la posibilidad de generar una reciprocidad positiva y solidaria en las relaciones interpersonales o sociales. Es en la interacción con los otros donde los comportamientos se construyen, vinculándose desde el principio con las redes de relaciones de la persona (Carr, 2016; Crespi \& Mikulic, 2009). Se incluye en el grupo de las capacidades para establecer vínculos interpersonales tales como el sentido del humor y la empatía (Auné, Abal, \& Attorresi, 2016a), y se encuentra en relación con la madurez moral (Casullo \& Fernández Liporace, 2007). Se ha comprobado que los efectos benéficos se extienden al actor de la conducta prosocial y hacia la sociedad entera (Aknin, Broesch, Hamlin, \& van de Vondervoort, 2015; Caprara, Alessandri, \& Eisenberg, 2012). El comportamiento prosocial 
es, sin dudas, un fenómeno complejo (Auné, Blum, Abal, Lozzia, \& Attorresi, 2014); para que se realice, interaccionan recíprocamente las características de la persona y de la situación (Penner \& Orom, 2010).

Dentro del contexto de estudio del comportamiento prosocial, resulta contrastante la gran cantidad de investigaciones acerca de la conducta prosocial en la infancia y en la adolescencia con los escasos desarrollos enmarcados en etapas posteriores. Se podría afirmar, así, que el estudio de dicho constructo en la adultez y la vejez es un campo incipiente, con una gran variedad de aristas por explorar (Auné et al., 2014).

En el plano internacional, para la medición de la conducta prosocial en adultos, se destaca la Escala para la Medición de la Prosocialidad en Adultos (Prosocialness Scale for Adults [PSA]) de Caprara, Steca, Zelli y Capanna (2005). Se trata de un instrumento breve, fácil de administrar y con potentes estudios psicométricos en relación con la teoría clásica. Sin embargo, el análisis de ítems realizado con el Modelo de Crédito Parcial Generalizado de la Teoría de la Respuesta al Ítem (TRI) reveló que mayoría de los parámetros de localización $\mathrm{b}$ hallados fueron negativos, mostrando que los ítems requirieron bajos niveles de prosocialidad para ser respondidos en forma favorable. Otros instrumentos para la medición de la conducta prosocial en la adultez son la Batería de Personalidad Prosocial (Penner, Fritzsche, Craige, \& Freifeld, 1995) y la Escala para la Medición de la Conducta Prosocial-Antisocial en el Ámbito Vital y en el Tráfico (López de Cózar, Esteban Martínez, Calatayud Miñana, \& Alamar Rocati, 2008).

A la escasez de instrumentos para la medición de la conducta prosocial en la etapa adulta, se suma la desactualización de la mayoría de ellos, y que el más novedoso, la PSA de Caprara et al. (2005), brinda un bajo nivel de información en niveles medio-altos y altos del rasgo, lo cual constituye una importante falencia. Estos factores denotan un evidente vacío en lo que respecta a la medición de la conducta prosocial en la etapa adulta.
Es por estos motivos que Auné, Abal y Attorresi (2016b), Auné y Attorresi (2017) y Auné (2018) han desarrollado un nuevo instrumento para la medición del comportamiento prosocial en la etapa adulta: la Escala de Conducta Prosocial (ECP), que evalúa el constructo a través de dos dimensiones: Ayuda y Confortar. Específicamente, la dimensión Ayuda permite medir la conducta prosocial en niveles relativamente elevados, aportando allí su máximo de información, lo cual se ha demostrado mediante la modelización de la ECP usando la TRI (Auné, 2018). Se han brindado asimismo otras evidencias de validez del instrumento a través de la correlación de cada una de las dimensiones de la ECP con la versión argentina de la Escala de Habilidades Prosociales de Morales Rodríguez y Suárez Pérez (Auné, Abal, $\&$ Attorresi, 2014).

\section{Objetivo e hipótesis}

Es consensuado que determinadas elecciones de carrera de nivel superior se vinculan con un mayor nivel de realización de conductas prosociales que otras. Por ejemplo, Bekkers (2006) y Hillygus (2005) hallaron mayores niveles de conducta prosocial en graduados de carreras con orientaciones sociales. La decisión de estudiar medicina, psicología o enfermería implica el conocimiento de que en el mismo ejercicio de estas profesiones se ayuda y conforta a otros; esto, muy posiblemente, se relacione con la prosocialidad. Se han obtenido evidencias de que la calidez y entusiasmo de los profesionales en su tarea de asistencia genera resultados más favorables en los pacientes (Martin-Raugh, Kell, \& Motowidlo, 2015).

Gámez y Marrero (2000) citan los siguientes motivos de ingreso a carreras universitarias: a) de logro, b) poder y prestigio, c) afiliativos, d) motivación por aprender o conocer, e) motivación por superar problemas personales y f) motivación extrínseca. Posteriormente, Caldera, Reynoso, González y Zamora, (2018) diseñaron la Escala de Motivos de Ingreso a las Carreras de 
Ciencias de la Salud (MICCS) basados en esta clasificación.

Recientemente, se han hallado resultados diversos en cuanto a las motivaciones para realizar una carrera vinculada con la salud. Por ejemplo, Mayta et al. (2015) encontraron dos motivaciones básicas en los estudiantes de medicina: aquella que buscaba el bienestar de los otros, y otra que perseguía el prestigio y las posibilidades económicas. Skatova y Ferguson (2014) se centraron en la motivación para estudiar diferentes carreras, hallando que en los estudiantes de medicina preponderaba el interés de ayudar, en combinación con el interés intrínseco en la carrera. El comportamiento prosocial se ha considerado predictivo del desempeño clínico de estudiantes de medicina norteamericanos (Kell, Motowidlo, Martin, Stotts, \& Moreno, 2014) e indios (Ghosh, Motowidlo, \& Nath, 2015).

Lo esperable sería que los estudiantes de carreras cuyo ejercicio involucra directamente a las conductas prosociales puntúen más alto que aquellos de carreras que no las involucran, en alguna o ambas dimensiones de la ECP. Por lo tanto, en el presente estudio se planteó como objetivo explorar la existencia de relaciones estadísticas entre el tipo de carrera que el participante está cursando \# relacionada directamente con la conducta prosocial o no\# y los puntajes en la ECP. Asimismo, se relevó la relación con la autopercepción explícita dada por el participante en conducta prosocial, así como en constructos relacionados (empatía, dadivosidad y altruismo). Adicionalmente, se exploró el posible mayor nivel de distorsión en la respuesta de alguno de los grupos.

\section{Método}

Diseño

La presente investigación constituye un estudio relacional y transversal. Las variables independientes no fueron manipuladas y, por lo tanto, no se encuadra en la categoría experimental (Montero \& León, 2007).

\section{Participantes}

Se solicitó la colaboración voluntaria de estudiantes universitarios de Argentina. La muestra estuvo compuesta por 1540 sujetos, de los cuales $79 \%$ fueron mujeres y $21 \%$ varones. La media de edad fue de 22.4 años, la mediana de 20 y la desviación estándar de 5.8. La edad varió entre 18 y 60 años. Las carreras con mayor peso en la muestra fueron psicología (29\%), medicina (10\%), abogacía (7\%), contaduría pública (7 $\%)$, licenciatura en administración de empresas (4.2\%) y licenciatura en artes audiovisuales (3.6\%). El resto de los sujetos se repartieron en más de 50 carreras diferentes. El $73.5 \%$ de los estudiantes relevados cursaba su carrera en la Universidad de Buenos Aires, el $18.9 \%$ en otras universidades nacionales y el $7.6 \%$ en universidades privadas. Fueron criterios de inclusión: ser residentes en Argentina, de entre 18 y 75 años, que cursaran en ese momento una carrera universitaria. Fueron criterios de exclusión la falta evidente de lucidez mental y la relación cercana entre los investigadores y los participantes.

\section{Instrumentos}

\section{Cuestionario sociodemográfico}

Se trata de una encuesta de relevamiento de datos sociodemográficos realizada ad hoc para el presente estudio, que incluye preguntas acerca de género, edad, carrera elegida, cantidad de materias aprobadas y universidad en la cual se cursa.

Escala de Conducta Prosocial ([ECP]; Auné, 2018; Auné, Abal, Eु Attorresi, 2016b; Auné Eु Attorresi, 2017)

Se compone de 15 ítems divididos en dos dimensiones: Ayuda (ocho ítems, con alfa de Cronbach de 0.77) y Confortar (siete ítems, con alfa de Cronbach de 0.79). Mientras que la dimensión Ayuda se refiere a comportamientos 
de asistencia, cuidado y compromiso con los otros, la dimensión Confortar se centra en acciones que manifiestan empatía, comprensión, refuerzo y soporte emocional. La modalidad de respuesta se especifica con una escala Likert de seis opciones que refleja la frecuencia con que se realiza la acción $(1=$ nunca, $2=$ casi nunca, $3=$ a veces, $4=$ con frecuencia, $5=$ casi siempre y $6=$ siempre). Se han aportado evidencias de validez a través de Análisis Factoriales Exploratorios y Confirmatorios. Asimismo, los reactivos se han modelizado mediante la TRI.

\section{Autopercepción de la Orientación Prosocial ([AOP]; Auné, 2018)}

Consta de cuatro enunciados, construidos ad hoc, que permiten obtener una autovaloración global del individuo respecto de su nivel de empatía, conducta prosocial, dadivosidad y altruismo. Con el fin de reducir ambigüedades en la compresión de cada aspecto, los sujetos recibieron una caracterización de cada constructo considerando que: a) una persona empática se pone en el lugar de otras personas, las comprende y experimenta sentimientos similares, apoyándolas emocionalmente; b) una persona prosocial realiza acciones que benefician a los demás; c) una persona dadivosa da, presta o comparte objetos, tiempo, dinero, experiencias, $\mathrm{u}$ otro aspecto de valor $\mathrm{y} d$ ) una persona altruista busca beneficiar a los demás aunque esto la perjudique. Posteriormente, se le solicitó al participante que se autovalorara en dichos rasgos, en una escala de 0 a 10. El alfa de Cronbach en esta investigación fue de 0.71 .

Escala Distorsión del Cuestionario de los Cinco Grandes (Big Five Questionnaire [BFQ]; Caprara, Borgogni, Barbaranelli, $\mathfrak{E}$ Moreno, 1995)

Está formada por 12 ítems que se contestan en una escala Likert de cinco opciones de respuesta (desde completamente falso para mí a completamente verdadero para mí). La escala de
Distorsión mide la deseabilidad social entendida como un estilo de respuesta que el sujeto puede poner en juego para mostrar aspectos favorables de su personalidad y eludir los menos favorables. Previamente a su aplicación, un grupo de expertos verificó la equivalencia lingüística y conceptual. En el manual de la adaptación, Bermúdez (1995) refiere un índice de consistencia interna alfa de Cronbach de 0.77. En la presente investigación, se halló un índice más bajo (alfa de Cronbach de 0.64), pero aceptable.

\section{Procedimiento}

En primer lugar, se depuraron los protocolos con más de $10 \%$ de respuestas incompletas y los outliers, mediante el cálculo de la distancia de Mahalanobis. El nuevo tamaño muestral, posterior a esta depuración, fue de 1383 participantes. Posteriormente, se dividió a los participantes en tres grupos según el tipo de carrera que estudiaban. Para ello, dentro de las carreras relacionadas con el comportamiento prosocial, se crearon dos categorías de acuerdo a la existencia de relación o no con la salud física. Se realizó esta distinción dado que en la bibliografía se han especificado resultados separadamente para estudiantes de medicina, psicología y otras carreras en relación con la conducta prosocial (p. ej., Ghosh et al., 2015; van Andel, Tybur, \& Van Lange, 2016), y con el fin de efectuar comparaciones de interés.

Grupo 1 (G1): carreras que implican conductas prosociales sin relación necesaria con la salud física: Licenciatura en Psicología, Psicopedagogía, Trabajo Social, Terapia Ocupacional, Musicoterapia y Minoridad. El tamaño de este grupo $\left(\begin{array}{ll}n & 1\end{array}\right)$ fue de 469 participantes.

Grupo 2 (G2): carreras que implican conductas prosociales en relación necesaria con la salud física: Medicina, Enfermería, Kinesiología, Fonoaudiología, Nutrición, Obstetricia y Odontología. El tamaño de este grupo $\left(n_{2}\right)$ fue de 192 participantes.

Grupo 3 (G3): carreras no incluidas en los Grupos 1 y 2, las cuales no involucran 
en forma directa a las conductas prosociales. El tamaño de este grupo $\left(\begin{array}{ll}n & 3\end{array}\right)$ fue de 722 participantes. De estos, las carreras con más proporción de estudiantes fueron Abogacía (13.4 \%), Contaduría Pública (12.7\%), Licenciatura en Administración (7.9\%), Licenciatura en Artes Audiovisuales (7.1\%), Recursos Humanos (5\%) e Ingeniería (4.7\%).

\section{Análisis de datos}

Dado que los puntajes en Ayuda y Confortar no se encuentran normalmente distribuidos (prueba de Kolmogorov-Smirnov, $p<0.0001$ para ambas dimensiones) se aplicó la prueba no paramétrica $\mathrm{H}$ de Kruskal-Wallis por separado para cada una de las dos dimensiones de la ECP en la muestra $(n=1383)$, empleando el programa SPSS versión 21. Además, para verificar la falta de normalidad, se analizó el gráfico de distribución y se obtuvieron los valores del índice de Mardia (1970) para la asimetría y curtosis multivariante con el macro para SPSS de DeCarlo (1997). Los resultados (coeficiente de asimetría de 33.38, $p$ $<0.00001$; coeficiente de curtosis de 21.62, $p<$ 0.001 ) mostraron que los datos no se distribuían de forma normal multivariante.

Para el contraste post hoc se utilizó la prueba no paramétrica $U$ de Mann-Whitney. El puntaje total de cada dimensión se adoptó como variable dependiente, mientras que el tipo de carrera se configuró como factor fijo. Se aplicó el mismo procedimiento para evaluar si existían diferencias en distorsión de respuesta entre los estudiantes de los tres grupos definidos y diferencias en el nivel de AOP. Se calculó para todas las pruebas de hipótesis la medida de tamaño del efecto $r$ de Rosenthal (Rosenthal, 1991).

\section{Resultados}

\section{Análisis descriptivo}

En cuanto al análisis descriptivo, en la Tabla 1, pueden apreciarse las medias y desviaciones estándar para cada dimensión de la ECP según el tipo de carrera (G1, G2 o G3). También se observan las medias y desviaciones estándar en AOP y en Escala de Distorsión del BFQ, según el tipo de carrera (G1, G2 o G3).

\section{Tabla 1}

Media y Desviación Estándar de Puntaje en la ECP, en la AOP y en la Escala de Distorsión del BFQ según Tipo de Carrera

\begin{tabular}{lrcc}
\hline \multirow{2}{*}{$\begin{array}{c}\text { Escalas y } \\
\text { dimensiones }\end{array}$} & \multicolumn{3}{c}{ Tipo de Carrera } \\
\cline { 2 - 4 } & $\mathbf{G 1}\left(\boldsymbol{n}_{\boldsymbol{l}}=\mathbf{4 6 9}\right)$ & $\mathbf{G 2}\left(\boldsymbol{n}_{\mathbf{2}}=\mathbf{1 9 2}\right)$ & $\mathbf{G 3}\left(\boldsymbol{n}_{\mathbf{3}}=\mathbf{7 2 2}\right)$ \\
\cline { 2 - 4 } & $\mathbf{M}(\boldsymbol{D E})$ & $\mathbf{M}(\boldsymbol{D E})$ & $\mathbf{M}(\boldsymbol{D E})$ \\
\hline Dimensión ECP & & & \\
Ayuda & $24.90(6.39)$ & $26.29(6.65)$ & $24.39(6.37)$ \\
Confortar & $30.76(5.15)$ & $31.57(6.06)$ & $29.97(5.96)$ \\
AOP & & & \\
Empatia & $7.72(1.66)$ & $7.65(1.90)$ & $7.45(1.87)$ \\
Conducta Prosocial & $6.38(1.80)$ & $5.97(2.29)$ & $5.71(2.15)$ \\
Dadivosidad & $7.19(1.71)$ & $7.30(1.83)$ & $6.78(2.05)$ \\
Altruismo & $5.28(2.29)$ & $5.21(2.68)$ & $4.99(2.50)$ \\
Distorsión & $23.94(5.75)$ & $26.33(5.00)$ & $25.57(5.28)$ \\
\hline
\end{tabular}

Nota. G1: Estudiantes de Lic. en Psicología, Psicopedagogía, Trabajo Social, Terapia Ocupacional, Musicoterapia y Minoridad, G2: Medicina, Enfermería, Kinesiología, Fonoaudiología, Nutrición, Obstetricia y Odontología, G3: Estudiantes de carreras que no involucran en forma directa a las conductas prosociales.

Análisis inferencial

Prueba no paramétrica de Kruskal-Wallis para la ECP

Los resultados de las pruebas $H$ de KruskalWallis indican que el tipo de carrera tiene efectos significativos sobre los puntajes tanto de la dimensión Ayuda $(p=0.001 ; r=0.082)$ como de la dimensión Confortar $(p=0.002 ; r=0.077)$.

En el caso de Ayuda, las comparaciones post hoc sobre el tipo de carrera mediante la prueba $U$ de Mann-Whitney señalan que los estudiantes de carreras pertenecientes a G2 obtienen puntuaciones significativamente superiores que los estudiantes de carreras pertenecientes a G1 $(p=0.016 ; r=0.057)$ y G3 $(p<0.0001 ; r=0.093)$. No existieron diferencias estadísticamente significativas entre los estudiantes de carreras de tipo G1 y G3 ( $p=$ $0.133)$.

Las comparaciones post hoc sobre el tipo de carrera para la dimensión Confortar señalan que 
los estudiantes de carreras pertenecientes a G1 y G2 no obtienen puntuaciones significativamente diferentes $(p=0.059)$. Además, los estudiantes de carreras pertenecientes a G1 y G2 obtienen puntuaciones significativamente superiores que los estudiantes de carreras pertenecientes a G3 $\operatorname{con} p=0.03(r=0.05)$ y $p=0.001(r=0.082)$, respectivamente.

Prueba no paramétrica de Kruskal-Wallis para la AOP

Los resultados de la prueba $H$ de Kruskal-Wallis para la AOP indican que el tipo de carrera tiene efectos significativos sobre el puntaje en Autopercepción de la Conducta Prosocial $(p$ $<0.001 ; r=0.093)$ y en Autopercepción de Dadivosidad ( $p=0.001 ; r=0.082)$. Por el contrario, el tipo de carrera no tuvo efectos estadísticamente significativos en la Autopercepción en Empatía $(p=0.064)$ y en la Autopercepción en Altruismo $(p=0.156)$.

Para los casos donde el efecto fue significativo, se realizaron comparaciones post hoc sobre el tipo de carrera mediante la prueba $U$ de Mann-Whitney. En cuanto a la Autopercepción en Conducta Prosocial, estas señalan que los estudiantes de carreras pertenecientes a G1 obtienen puntuaciones significativamente superiores que los estudiantes de carreras pertenecientes a G3 $(p<0.001 ; r=0.093)$. No existieron diferencias estadísticamente significativas entre los estudiantes de carreras de tipo $\mathrm{G} 1$ y $\mathrm{G} 2(p=0.054)$ y entre $\mathrm{G} 2$ y $\mathrm{G} 3$ $(p=0.101)$. Mientras que no hubo diferencias significativas entre G1 y G2 $(p=0.275)$ con respecto a la Autopercepción en Dadivosidad, sí se diferenciaron $\mathrm{G} 1$ y G3 ( $p=0.003 ; r=0.073)$, así como G2 con respecto a G3 $(p=0.002 ; r=$ 0.077).

Prueba no paramétrica de Kruskal-Wallis para la Escala de Distorsión del BFQ

Los resultados de la prueba $H$ de Kruskal-Wallis para la Escala Distorsión del BFQ indican que el tipo de carrera tiene efectos significativos sobre el puntaje con $p<0.0001$. Las comparaciones post hoc sobre el tipo de carrera mediante la prueba $U$ de Mann-Whitney señalan que los estudiantes de carreras pertenecientes a G1 obtienen puntuaciones significativamente inferiores que los estudiantes de carreras pertenecientes a G2 $(p<0.0001 ; r=0.093)$ y G3 $(p<0.0001 ; r=0.093)$. No existieron diferencias estadísticamente significativas entre los estudiantes de carreras de tipo G2 y G3 ( $p=$ 0.089).

\section{Discusión}

Los resultados son coherentes con la hipótesis planteada de que los estudiantes universitarios de profesiones que implican, por su ejercicio futuro, solidaridad, ayuda o conductas empáticas hacia otros, puntuarán significativamente más alto en la ECP que aquellos estudiantes de carreras que no involucren en forma directa una conducta prosocial.

Los estudiantes de medicina y carreras afines manifestaron un alto nivel de conductas prosociales tanto en Ayuda como en Confortar, con diferencias muy significativas con respecto a los estudiantes de carreras muestreadas que no involucran directamente dichas conductas. En Ayuda, se diferenciaron incluso de los estudiantes de las carreras de G1, aunque mostraron mayores niveles de distorsión que estos últimos. Concordantemente, van Andel et al. (2016) hallaron que, en una muestra de estudiantes universitarios holandeses, quienes se estaban formando en medicina y en psicología tuvieron actitudes más positivas hacia la donación de órganos que sus pares de de ciencias económicas, siendo los estudiantes de medicina, los de mayor puntaje.

Los estudiantes de Licenciatura en Psicología y carreras afines, tal como era esperable, tuvieron significativamente mayores puntajes en la dimensión Confortar que los estudiantes de carreras muestreadas que no involucran directamente las conductas prosociales y un nivel similar que los estudiantes de G2. 
Es decir, los estudiantes de G1 y G2 tuvieron similares niveles en su capacidad de expresión empática, de cuidado y comprensión, a los otros. Estos resultados son coherentes dada la interacción social particular que necesariamente involucran. Pero en la dimensión Ayuda, fue mayor el nivel de los estudiantes de G2. El hecho de estar en contacto directo con enfermedades de características físicas puede implicar un riesgo mayor (por ejemplo, de contagio) o un sacrificio más grande para su práctica que las carreras de G1.

Es así como podría ser necesario un mayor nivel de conducta prosocial para elegir estudiar las carreras de G2. Esto resulta coincidente con numerosas investigaciones donde se ha hallado que las razones humanitarias son determinantes para decidir estudiar Medicina (p. ej., PadillaCuadra, Vindas-Sánchez, \& Villalobos-Pérez, 2012). Se ha puntualizado que el razonamiento moral es un elemento determinante para la formación médica (Escobar-Melo, Díaz Amado, Páramo Cely, Suárez Obando, \& León Guevara, 2010). Los valores de autotrascendencia, que enfatizan la disminución de la importancia dada al sí mismo y la atención centrada en los otros y en la naturaleza, se encuentran positivamente relacionados con las conductas prosociales (Boer \& Fischer, 2013; Caprara et al., 2012). Los resultados concuerdan asimismo con lo hallado por los autores cuyo marco teórico fue asentado en la introducción: Bekkers (2006), Hillygus (2005), Mayta et al. (2015) y Skatova y Ferguson (2014).

En relación con la autopercepción explícita, la misma influye en los resultados obtenidos. En este caso, el tipo de carrera tuvo efectos significativos sobre el puntaje en Autopercepción de la Conducta Prosocial y en Autopercepción de Dadivosidad, pero no así en la Autopercepción en Empatía y Altruismo. Se puede observar cómo la diferencia en la medición da lugar a resultados diversos sobre la misma muestra, aunque el mayor puntaje de las carreras de la salud en su percepción como más prosociales y dadivosos resulta concordante con los resultados de la ECP.

Estos resultados se pueden relacionar con el concepto de altruismo. Si bien toda conducta altruista es prosocial, no es así a la inversa (González Portal, 2000). Para que una conducta sea considerada altruista, existen requisitos exigentes: implicar más perjuicios que beneficios, priorizar la necesidad de los otros e incluir sacrificio (Howard \& Piliavin, 2000). Se ha argumentado que el comportamiento altruista puro requiere la inhibición de los impulsos egoístas, y esto se encuentra esencialmente relacionado con la capacidad empática. La empatía involucra aspectos emocionales, cognitivos y motivacionales (Decety \& Cowell, 2018). Los resultados concuerdan con lo hallado por los autores cuyo marco teórico fue asentado en la introducción: Bekkers (2006), Hillygus (2005), Mayta et al. (2015) y Skatova y Ferguson (2014).

En cuanto a las limitaciones de la presente investigación, se encuentra principalmente el tipo de muestreo (por accesibilidad) y el desbalance de los tamaños muestrales de cada uno de los grupos. Se ha estudiado la deseabilidad social, que fue mayor para el grupo de carreras relacionadas con la salud física y no vinculadas con la conducta prosocial. Esto podría estar distorsionando las respuestas, por lo que se requiere un análisis más minucioso.

En futuras investigaciones, se podría modificar el criterio de conformación de los grupos, identificando, por ejemplo, carreras cuyo desempeño involucre beneficiarios no humanos. $\mathrm{Al}$ estar la muestra circunscripta a estudiantes universitarios, los resultados obtenidos pueden ser relevados en estudiantes de otros niveles educativos. Una muestra más heterogénea permitirá incrementar la generalización de resultados, además de realizar comparaciones entre las motivaciones y el grado de comportamiento prosocial en diferentes niveles educativos. Por otra parte, sería conveniente estudiar en este rasgo las condiciones para la existencia de estabilidad a lo largo de los ciclos vitales. Especialmente, sería importante estudiar la relación entre los niveles de conducta prosocial y los rasgos de personalidad. En futuros desarrollos, podría asimismo ser de gran utilidad la construcción de instrumentos de medición específicos de la conducta prosocial en la adultez 
para distintos ámbitos, tales como el clínico, el jurídico, el educacional y el laboral.

\section{Agradecimientos}

Esta investigación fue financiada con los subsidios de la Universidad de Buenos Aires UBACyT 2018 con Códigos 20020170100200BA y 20020170200001BA y de la Agencia Nacional de Promoción Científica y Tecnológica (ANPCyT) PICT-2017-3226.

\section{Referencias}

Aknin, L. B., Broesch, T., Hamlin, J. K., \& van de Vondervoort, J. W. (2015). Prosocial behavior leads to happiness in a smallscale rural society. Journal of Experimental Psychology: General, 144(4), 788-795. https ://doi.org/10.1037/xge0000082

Auné, S. E. (2018). Construcción de un test de comportamiento prosocial y su modelización con la Teoría de la Respuesta al Ítem (Tesis doctoral inédita). Facultad de Psicología de la Universidad de Buenos Aires. Recuperado de http://kohamigracion.psi.uba.ar/cgi-bin/ koha/opac-detail.pl?biblionumber $=53412$ \%20thumbnail-shelfbrowser

Auné, S. E., Abal, F. J. P., \& Attorresi, H. F. (2014). Versión argentina de la Escala de Habilidades Prosociales de Morales Rodríguez y Suárez Pérez (2011). Praxis. Revista de Psicología, 16(26), 31-48. Recuperado de https://ri.conicet.gov.ar/bitstream/handl e/11336/52577/CONICET_Digital_Nro.ee

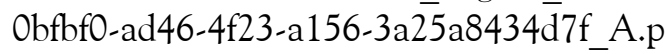
$\mathrm{df}$ ? sequence $=2$

Auné, S. E., Abal, F. J. P., \& Attorresi, H. F. (2016a). Antagonismos entre concepciones de empatía y su relación con la conducta prosocial. Revista de Psicología, 17(2), 137-149. https://doi.org/10.18050/revpsi.v 17n2a7.2015

Auné, S., Abal, F. J. P., \& Attorresi, H. F. (2016b). Diseño y construcción de una escala de conducta prosocial para adultos. Revista Iberoamericana de Diagnóstico y Evaluación Psicológica, 42 (2), 15-25. https://doi.org/10. 21865/RIDEP42 15

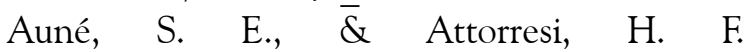 (2017). Dimensionalidad de un test de conducta prosocial. Revista Evaluar, 17(1), 29-37. https://doi.org/10.35670/1667-4545 .v17.n1.17072

Auné, S. E., Blum, G. D., Abal, F. J. P., Lozzia, G., \& Attorresi, H. F. (2014). La Conducta Prosocial: estado actual de la investigación. Perspectivas en Psicología, 11(2), 21-33. Recuperado de http://www.seadpsi.com.ar/ revistas/index.php/pep/article/view/153

Bekkers, R. (2006). Traditional and health related philanthropy: The role of resources and personality. Social Psychology Quarterly, 68(4), 349-366. Recuperado de https://ww w.asanet.org/sites/default/files/savvy/image s/journals/docs/pdf/spq/Dec06SPQFeature. pdf

Bermúdez, J. (1995). Manual del Cuestionario "Big Five" (BFQ). Madrid: TEA Ediciones.

Boer, D., \& Fischer, R. (2013). How and when do personal values guide our attitudes and sociality? Explaining cross-cultural variability in attitude-value linkages. Psychological Bulletin, 139, 1113-1147. http s://doi.org/10.1037/a0031347

Caldera, J., Reynoso, O., González, S., \& Zamora, M. (2018). Propiedades psicométricas de un instrumento de motivos de ingreso a carreras. Revista de Docencia Universitaria, 16(1), 33-49. https://doi.org/10.4995/redu. 2018.6052

Caprara, G. V., Alessandri, G., \& Eisenberg, N. (2012). Prosociality: The contribution of traits, values, and self-efficacy beliefs. Journal of Personality and Social Psychology, 102 (6), 1289-1303. https://doi.org/10.1037 /a0025626

Caprara, G. V., Borgogni, L., Barbaranelli, C., \& Moreno, J. B. (1995). BFQ: Cuestionario "Big Five": manual. TEA Ediciones.

Caprara, G. V., Steca, P., Zelli, A., \& Capanna, C. (2005). A new scale for measuring adults' prosocialness. European Journal of 
Sofía Esmeralda Auné, Facundo Juan Pablo Abal, Horacio Félix Attorresi.

Psychological Assessment, 21(2), 77-89. http s://doi.org/10.1027/1015-5759.21.2.77

Carr, A. (2016). The handbook of child and adolescent clinical psychology. A contextual approach (3. ․ ed.). Londres: Routledge.

Casullo, M. M., \& Fernández Liporace, M. (2007). Las propuestas de la psicología positiva: iuniversales psicológicos $\mathrm{O}$ particulares de una visión cultural? Anuario de Investigaciones, 14, 261-268. Recuperado de https://www.redalyc.org/service/redalyc/ downloadPdf/3691/369139943022/6

Crespi, M. C., \& Mikulic, I. M. (2009). Reinserción social de liberados condicionales: análisis de la dimensión relacional desde el enfoque de redes de apoyo social. Anuario de Investigaciones, 16, 211-221. Recuperado de https://www.redal yc.org/pdf/3691/369139945070.pdf

DeCarlo, L. T. (1997). On the meaning and use of kurtosis. Psychological Methods, 2(3), 292-307. https://doi.org/10.1037/1082-989 X.2.3.292

Decety, J., \& Cowell, J. M. (2018). Empathy and its relationship to moral behavior. En A. R. Beech, A. J. Carter, R. E. Mann \& P. Rotshtein (Eds.), The Wiley Blackwell handbook of forensic neuroscience (pp. 145-165). New Jersey: Wiley-Blackwell.

Donaldson, S. I., Dollwet, M., \& Rao, M. A. (2015). Happiness, excellence, and optimal human functioning revisited: Examining the peer-reviewed literature linked to positive psychology. The Journal of Positive Psychology, 10(3), 185-195. https://doi.org/ 10.1080/17439760.2014.943801

Escobar-Melo, H., Díaz Amado, E., Páramo Cely, L. C., Suárez Obando, F., \& León Guevara, C. (2010). Caracterización del razonamiento moral en la práctica clínica en relación con el proceso de formación médica en una Facultad de Medicina, categoría altruismo y dignidad, beneficencia, confidencialidad y deber de cuidado. Revista Colombiana de Bioética, 5(1), 34-56. Recuperado de https://www.redalyc.org/service/redalyc/ downloadPdf/1892/189217244003/1
Gámez, E., \& Marrero, H. (2000). Metas y motivos en la elección de carrera de psicología. Revista Electrónica de Motivación y Emoción, 3(5-6). Recuperado de http://reme.uji.es/articulos/agomee1071 912100/texto.html

Ghosh, K., Motowidlo, S. J., \& Nath, S. (2015). Technical knowledge, prosocial knowledge, and clinical performance of Indian medical students. International Journal of Selection and Assessment, 23(1), 59-70. https://doi.or g/10.1111/ijsa.12095

González Portal, M. D. (2000). Conducta prosocial: evaluación e intervención. Madrid: Morata.

Hillygus, D. S. (2005). The missing link: Exploring the relationship between higher education and political engagement. Political Behavior, 27(1), 25-48. https://doi. org/10.1007/s11109-005-3075-8

Howard, J. A., \& Piliavin, J. A. (2000). Altruism. Encyclopedia of sociology. Nueva York: Macmillian.

Kell, H. J., Motowidlo, S. J., Martin, M. P., Stotts, A. L., \& Moreno, C. A. (2014). Testing for independent effects of prosocial knowledge and technical knowledge on skill and performance. Human Performance, 27, 311-327. https://doi.org/10.1080/08959 285.2014.929692

López de Cózar, E., Alonso Plá, F., Esteban Martínez, C., Calatayud Miñana, C., \& Alamar Rocati, B. (2008). Diseño y validación de una escala para la medición de la conducta prosocial-antisocial en el ámbito vital $y$ en el tráfico. Valencia, ES: Instituto Universitario de Tráfico y Seguridad Vial. Recuperado de https://www.uv.es/metras/docs/2008_en cuestas_lopez_de_cozar_2_acta.pdf

Macbeth, G., Cortada de $\bar{K}$ ohan, N. C., \& Razumiejczyk, E. (2007). Perspectivas en epistemología de la psicología. Psicología y Psicopedagogía, 16(1), 1-15. Recuperado de https:/www.researchgate.net/profile/Guill ermo Macbeth/publication/228754866 P ERSPECTIVAS_EN_EPISTEMOLOGIA 
DE_LA_PSICOLOGIA/links/Ofcfd511f1 $\overline{3} 3 \mathrm{fb} \bar{b} a \mathrm{a} 8 \overline{0} 00000 . \mathrm{pdf}$

Macbeth, G., Cortada de Kohan, N. C., Razumiejczyk, E., \& López Alonso, A. O. (2006). La investigación científica en psicología: un desarrollo histórico. Psicología y Psicopedagogía, 5(15), 1-17. Recuperado de https://racimo.usal.edu.ar/4604/1/12834611-1-PB.pdf

Mardia, K. V. (1970). Measures of multivariate skewnees and kurtosis with applications. Biometrika, 57(3), 519-530. https://doi.org/ 10.1093/biomet/57.3.519

Marean, C. W. (2015). La especie más invasora. Investigación y Ciencia, 469, 14-21. Recuperado de https://dialnet.unirioja.es/se rvlet/articulo? codigo $=5203392$

Martin-Raugh, M. P., Kell, H. J., \& Motowidlo, S. J. (2015). Prosocial knowledge mediates effects of agreeableness and emotional intelligence on prosocial behavior. Personality and Individual Differences, 90, 41-49. https://doi.org/10.1016/j.paid.2015. 10.024

Mayta, P., Mezones, E., Carbajal, D., Pereyra, R., Montenegro, J., Mejía, C., ... Grupo Colaborativo Latinoamericano para la Investigación en Recursos Humanos en Salud (Red-LIRHUS). (2015). Validación de una escala para medir las Motivaciones para estudiar Medicina (MEM-12) en estudiantes Latinoamericanos. iMedPub Journals, 11(3-7), 1-7. https://doi.org/10.38 23/1257

Mikulic, I. M., Muiños, R., Crespi, M., Fernández, G., Monges, M. E., Lorenzut, V., \& Ojeda, G. (2006). "Transiciones ecológicas": estudio del afrontamiento de las crisis vitales. Migración, encarcelamiento y divorcio desde el enfoque de la psicología positiva. Anuario de Investigaciones, 13, 175-184. Recuperado de https://www.redalyc.org/pdf 13691/369139942017.pdf

Montero, I., \& León, O. G. (2007). Sistema de clasificación del método en los informes de investigación en psicología. International Journal of Clinical and Health
Psychology, 5(1), 115-127. Recuperado de https://www.redalyc.org/service/redalyc/ downloadPdf/337/33701007/2

Padilla-Cuadra, J. I., Vindas-Sánchez, L., \& Villalobos-Pérez, A. (2012). Decision to study medicine: Determinants and specialty choice. Acta Médica Costarricense, 54(2), 109-113. Recuperado de http://www.redaly c.org/pdf/434/43422619007.pdf

Penner, L., Fritzsche, B., Craiger, J., \& Freifeld, T. (1995). Measuring the prosocial personality. En J. Butcher \& C. Spielberger (Eds.), Advances in Personality Assessment (Vol. 10, pp. 147-163). Hillsdale, NJ: Erlbaum.

Penner, L. A., \& Orom, H. (2010). Enduring goodness: A person-by-situation perspective on prosocial behavior. En M. Mikulincer \& P. R. Shaver (Eds.), Prosocial motives, emotions, and behaviors: The better angels of our nature (pp. 55-72). Washington, DC: American Psychological Association.

Rosenthal, R. (1991). Meta-analytic procedures for social research (2.. ed.). Newbury Park, CA: Sage.

Schroeder, D. A., \& Graziano, W. G. (2015). The field of prosocial behavior: An introduction and overview. En D. A. Schroeder \& W. G. Graziano (Eds.), The Oxford handbook of prosocial behavior (pp. 3-36). Nueva York: Oxford University Press.

Seligman, M. E. P., \& Csikszentmihalyi, M. (2000). Positive psychology: An introduction. American Psychologist, 55(1), 5-14. https://doi.org/10.1037/0003-066X.5 5.1.5

Seligman, M. E., Parks, A. C., \& Steen, T. (2004). A balanced psychology and a full life. Philosophical Transactions-Royal Society of London Series B: Biological Sciences, 359, 1379-1382. https://doi.org/10.1098/rstb.20 04.1513

Skatova, A., \& Ferguson, E. (2014). Why do different people choose different university degrees? Motivation and the choice of degree. Frontiers in Psychology, 5, 1244. ht tps://doi.org/10.3389/fpsyg.2014.01244 
Sofía Esmeralda Auné, Facundo Juan Pablo Abal, Horacio Félix Attorresi.

van Andel, C. E., Tybur, J. M., \& Van Lange, P. A. (2016). Donor registration, college major, and prosociality: Differences among students of economics, medicine and psychology. Personality and Individual Differences, 94, 277-283. https://doi.org/10. 1016/j.paid.2016.01.037

\section{Notas}

* Artículo de investigación. 